\title{
Plants as a natural resource of bioactive compounds for drug formulation to control infectious diseases
}

\author{
Jawaid Akram¹, Waseem Ahmed ${ }^{* 2}$ Rafia Azmat $^{1}$ \\ Department of Agriculture ${ }^{2}$, University of Haripur, Pakistan \\ Department of Chemistry, University of Karachi \\ Corresponding Author's Email: waseemuaf12@gmail.com
}

\begin{abstract}
In recent years, plants derived phytochemicals attracting interest of researchers due to the antimicrobial/antibacterial resistance of the antibiotic drugs against several diseases; as medications from medicinal plants are safe, effective, easily available and infrequently accompanied. This review, highlighted significance of the secondary metabolites and their mode of action. Drug discovery using natural products is a challenging task for designing new leads. It describes the bioactive compounds derived from natural resources, its phytochemical analysis, characterization and pharmacological investigation. It focuses on the success of these resources in the process of finding and discovering new and effective drug compounds that can be useful for controlling infectious diseases. From many years, natural products have been acting as a source of therapeutic agents and have shown beneficial uses. The phytochemical screening of several plants using advanced analytical techniques showed that plants act as resource of antioxidant, antibacterial, antiviral, antiinflammatory compounds which can take the place of modern medications in controlling microbial diseases. Furthermore; The extract of plant's leaves, root or stem showed high scavenging properties related to free radical generation. It was concluded that only natural product drug discovery may plays an important role for formulation of new drugs to develop the scientific evidence of these natural resources.

Current review highlighting the significance of the plant derives bioactive compounds and their action against different diseases.
\end{abstract}

Keywords: Phytochemicals; resistance; bioactive compounds; scavenging

\section{Highlights:}

$>$ Significance of plants in controlling diseases

$>$ bioactive compounds as an antioxidant

$>$ plants extract as an anti-biological agent

\section{Introduction}

\subsection{Significance of herbal Medicinal plants in the current era as biomedicines}

Throughout the initial time of the world, plant natural products or bioactive compounds found to be act as a traditional medication, therapies, tonics and oils deprived of any acquaintance about the bioactive compounds present in plants which were based only on consideration of their effect rather than any experiment (Bernardini, et al., 2018). The knowledge about traditional medicines based on plants required much attention for the new lead formulation due to the side reactions of modern medications. The recent advancements in drugs discovery with the development of the antibiotic and their uses against chronic diseases are effective for the time beings, while with the passage of time their side effects, can leads to some non-curable mechanisms of diseases, which raise the question on the effectiveness of such medicine followed by the alternative medicine for complete and long-lasting cure (Dulger \& Gonuz, 2005). During this present advancement of the world researches (Pharmacist, Chemist and Phyto scientists) showing their interest to search those compounds from herbal resources which showed no side effects and long lasting or durable for human health under chronic as well as acute diseases (Calixto, et al.,1998; Muthukumran, et al., 2011). Herbal plants provided a vast variety of active compounds for clinical use 
and also gives the thought for synthesis of these compounds which require continuous experimental, analytical and currently computational analysis for their testing as an effective medicine.

\subsection{Plants as Natural resources of bioactive compound}

Plants are the significant services provider to human from environmentally friendly sustainable life including food to medicine as well as like respiration, climate, global warming or as potential source in pharmaceutical and other allied applications. Plants comprises of numerous dynamic compounds likewise phenols, alkaloids, tannins, glycosides, fixed oils, steroids, volatile oils, and flavonoids, found in their particular parts of the plants such roots, barks, blossoms, seeds, natural products, etc.

Human body well-arranged by antioxidant defense framework or system that is utilize and develop to stop antioxidant activities through bioactive chemicals like superoxide dismutase, glutathione peroxidase, and catalase. Some plants also contained non-enzymatic cancer avoidance operators or preventing agents like, vitamins E, Vitamins C \& glutathione, thiol cancer avoidance specialists, melatonin, carotenoids.

About all living creatures have antioxidant as well as repair mechanisms toward securing themselves against their oxidative stress, upset or hurt, hence all living beings have antioxidant and repair system or mechanism to secure themselves against oxidative harm or damage, but these frameworks property do not totally anticipate harm or damage in a few case so, this lack may result in oxidative push or stress, in any case this oxidative stress or push produced by free radicals in association to the improvement of numerous illnesses in which aggravation plays an critical part, such as cardiovascular infections, inveterate obstructive pneumonic infections, neurodegenerative rheumatoid pain, asthma, some immune system infections, and a few cancers (Allegra, 2019).

\subsection{Active Role of antioxidant in human body}

Antioxidants compounds are constituents that may stop or slow injury to cells triggered by free radicals, unstable molecules that the body generated as a reaction to environmental biotic and abiotic stress. Oxidation processes in the human body can damages the various cell membranes and some other compositions, like cellular proteins, lipids, DNA etc. During this process oxygen is metabolized and convert into the uncertain and non-stable molecules refereed as free radicals, adversely affect or steal electrons from other molecules, which may cause the serious damage to DNA and cell activities.

To a few free radicals, the body can adapt, and needs them to work viably. Whatever it may be, the harm or damage caused by overloading or burdening of free radicals over time may end up irreversible, leading to certain heart, lung, kidney, and liver disease infections and a few cancers such as oesophageal, stomach, digestive, and oral chronic diseases. Heat, smoking, alcohol, heat, pollution, stress and other variables may promote oxidation.

\subsection{Antioxidants activity and free radicals'generations}

Antioxidant was introduced in 1990s when researcher start to study the free radical damaged was encompass in arteryclogging atherosclerosis. The antioxidant can be synthesized or found naturally. Some herbal plants or food prepared by plants act as a rich source of the antioxidant which are referred plants phytochemicals or phytonutrients. While herbal plants contain some bioactive compounds having antioxidant activity that can prevent damages caused by free radicals through neutralizing them, like nutrient antioxidants, vitamins A, C and E, and minerals including certain copper, zinc and selenium. In plants, the phytonutrients have greater antioxidant potential than vitamins or minerals. These are called the non-nutrient 
antioxidants and include lycopenes in tomatoes and anthocyanins found in cranberries etc. These free radicals are harmful to the body due to which degradation of essential compound takes place that contributes to loss of vision or eye damage, inflammation or stiffness of the joints (arthritis), disruption to nerve cells or central nervous System (CNS) in the brain, which leads to several disorders in human body (Adil, 2006).

\subsection{Antioxidants species of plants to combat numerous bacterial infections}

Antioxidants compounds from plants have the ability to fight numerous bacterial and viral infections in human body and reduce the chances of several prevalent diseases like heart disease clogging or certain forms of cancer by scavenge free radicals from the body cells and inhibit or reduce the harmful effects by oxidation (Khameneh et al., 2019).

Phytochemical species are generally suggested globally by scientists, for example, someone who took quite a lot of antioxidant lycopene by taking tomatoes would be protected from the prostate and ovarian cancers, corn and broccoli has lutein that serves to protect the eyes and their vision of sustainability, flavonoids reveal in green tea and also discovered in fruits, vegetables, grains, bark, roots, stems, flowers. Flavonoids are associated with a wide range of health-promoting effects and is an essential component in a variety of nutraceuticals. Pharmaceutical, medicinal and cosmetic applications, because of their antioxidative, anti-mutagenic, anti-carcinogenic and anti-inflammatory properties. Yahia et al., (2020) observed the radical scavenging potential of extract of leaves, fruits, and seed of Z. lotus and Z. mauritiana; showed the presence of highest phenolic compounds, which was validated through LC-ESI-MS analysis. They reported 28 bioactive compounds irrespective of parts and species of the both plants which found to be active against microbial species including Staphylococcus aureus, Listeria monocytogenes, Salmonella typhimurium and Escherichia coli.

\subsection{Plants as meditative or therapeutic products or antioxidants}

The pragmatic application of plants in the curing of sicknesses is an extensive exercise since earliest times. The therapeutic potential of herbal plants found in different region of the world is famous as antibacterial, antioxidant and antiviral agents against bacterial diseases which is mainly related with the bioactive compounds found in their extracts. These extracts may contain several compounds like flavonoids, anthocyanins, carotenoids, dietary, glutathione, organic vitamins and reactive or volatile or endogenous metabolites, with significant antioxidant potential (Subedi, et al., 2014).

Therefore, such findings are focused towards naturally occurring bioactive compounds through sources including seeds of certain plants, as chemical compounds implicated throughout the reduction of cellular oxidative stress, may well have been segregated from plant extracts and then used it to develop drugs used in the treatment and prevention of several various cancers (Atala et al., 2009). Antioxidants derived from plants can operate effectively as quenchers of single and triple oxygen species, decomposers of peroxides, inhibitors of the enzymes and synergists. (Choi et al..2002). World Wellbeing Organization (WHO) estimates that $80 \%$ of populations in developed nations dependent on traditional and conventional pharmaceuticals for their basic well-being, care, treatment, and nearly $85 \%$ of drug treatments require the use of plant extracts. This means that approximately 3.5 to 4 billion people worldwide rely on plants as medication sources (de Laet Santana et al., 2016).

These biochemicals, such as polyphenols, resins, hormones, glycosides, volatile or reactive oils, tannins, fixed oils, and flavonoids, and phenols, explicitly repress the function of natural targets (Gupta, et al., 2012). Nevertheless, characteristic things (and their derivatives and analogs) already refer to over $50 \%$ of all pharmaceutical goods in clinical use, with low natural products originating from plants contributing to $25 \%$ of the overall. A few significant examples are Galega officinalis L. by galegine, which was the display for the amalgamation of metformin and other antidiabetic drugs; Papaver somniferum L. by papaverine, which created the premise for verapamil, utilized in the treatment of hypertension. Opium, tranquilizer drug, gained from the unripe seedpods of the opium poppy. The utilization of the plants for therapeutic purposes 
declares to the largest use of biodiversity within the world. Numerous species of plants are utilized as medications rather than for nutrition (Nascimento, et al., 2000).

\subsection{Bioactivity / Bio-affinity Profiling for drugs and other formulation processes}

Multiple-drug susceptibility in human pathogenic microorganisms has evolved over the course of time in the context of the ineffectual usage of synthetic antimicrobial drugs. As they are commonly used for the treatment of persistent pathogens and other diseases. Research or study into biologically reactive or active extracts and compounds from characteristic resources like herbal or medicinal plants has therefore been of significant interest to researchers inside. An attempt has been made to discover new sources for drugs that should be valuable in combating infectious diseases. Literature search revealed that the solvent extract of medicinal plants used for screening of antimicrobial screening since long time (Sokmen, et al., 1999; Keleş, et al., 2001; Erdogrul, 2002; Dulger \& Gonuz, 2005 \& Ercisli, 2007). However, antibiotics are also correlated with adverse effects such as hypersensitivity, allergic reaction, and immune suppression. (Davies, 1994; Sokmen, et al., 1999; Ozturk \& Ercisli, 2005; Salehi, et al., 2005).

Several species of Ziziphora (Labiatae) and Thymus (Labiatae) are popularly used it to combat infectious diseases and microbial spices (Baytop, 1984; Sokmen, et al., 2004; Ismaili, et al., 2004). Hypericum (Guttiferae) species are widely used in traditional medicine for the healing of wound infections (Baytop, 1984). Herbal medicines of both natural and cultivated sources are being used popularly in food industry, conventional medicine and also for their flavor enhancers characteristics on a significant basis (Baytop, 1984).

Bioactivity shows the property of a compound or a set of compounds to regulate biochemical and physiological capacities of living beings. Characteristic verification of bioactive compounds in mixtures, such as common extracts or metabolic blends, is a critical challenge in numerous logical and application areas that are frequently profoundly tangled. Most of the times, bioactive compounds are display in exceptionally complex mixtures. These mixtures can at the same time contain different bioactive compounds with diverse components of activity or with synergistic and/or opposing impacts. Bioactivity profiling of an extract of compounds implies bioactivity appraisal of extract constituents and distinguishing proof of conceivably. Bioactivity profiling is for the most part coordinated at finding bioactive for a specific target, most frequently a drug target. Commonly, it is utilized in several screening programs that point at the distinguishing proof of bioactive compounds from diverse test sources. The antimicrobial activity of the extract of three plants Duranta plumieri variegata, Lantana camara, and Citharexylum spinosum in chloroform showed the presence of bioactive compounds which were proved by GC/MS techniques (Ashmawy et al., 2018).

For illustration, in endeavor to find modern lead compounds, pharmaceutical industry as well as academic groups interested about medicated disclosure in managing with examination of different bioactive compounds in extract to control diseases which require full engineered or furnish collections of instruments with common items. The examination of metabolic extract of drugs created either in vivo or in vitro for potential bioactive metabolites expresses to another exceptionally imperative application of bioactivity profiling in dignified disclosure (Zheng \& Dong 2011). Moreover, bioactivity profiling of mixtures is imperative in toxicology, forensics, natural and nourishment examination (Xiao, et al., 2017).

Microbes are the most seasoned shapes of life on soil, they are strikingly different and exist in astonishing numbers. Microscopic organisms are gathering of micro-organisms that are single cells and roughly one micron in transverse distance across. Infections caused by microbes include a few of the foremost common diseases within the world, it is considered one of the debilitating issues in therapeutic field, past, current, and likely future (Relman, 2002). Microbes have been classified compatible to phenotype, counting shape, estimate, recoloring properties and biochemical properties. As of late, the 
classification has been overwhelmed by genotype investigation, particularly utilizing preserved parts such as 16S ribosomal RNA. A few species of microbes, such as Pseudomonas aeruginosa, are opportunistic pathogens and cause infection basically in individuals enduring from immunosuppression (Entenza, et al., 2014).

Diseases that are triggered by microscopic organisms are treated with drugs called anti-microbials or antibiotic, sometime showed side reaction or microbes showed resistance against antibiotic (Sommer \& Dantas, 2011). Bacterial resistance to anti-microbial drugs is one of the foremost genuine dangers to worldwide open wellbeing and the issue knows no boundaries. Drug-resistant microbes of all sorts can move among individuals and creatures, from one nation to another without notice. The issue is clearly more extreme in creating nations where medicate accessibility, is limited and resistance is elevated (Breu, et al., 2008). The World health organization (WHO) describes antibiotic resistance as a serious threat that's not predictable (https://www.who.int/news-room/fact-sheets/detail/antibiotic-resistance). The modern multi-drug pathogenic strains which assembling huge numbers of resistance components, significantly limiting therapeutic alternatives. Moreover, the enormous money related and calculated burden on health care sectors over the globe (Wright, 2012). Ultimately, our essential concern with respect to resistance is that resistant bacteria are more difficult to treat with the passage of time while contaminations caused by them increases with respect to time. In pharmacognosy studies, natural compounds, which are primarily determined from plants, have been broadly misused as a resource in medication advancement. Continuous search and investigation on plant is still require in current modern era as bacteria are also modernized in term of their resistance against antibiotics (Ozturk, \& Ercisli, 2005).

\subsection{Challenges and opportunities related to medicinal plants}

Plants have conventionally been used as a source of bioactive compounds in the drug discovery since long time and act as source of the medicine for treatment of infectious diseases including Cancer too. Furthermore, the history of mankind is full with the use and misuse of bioactive compounds of plants. The new drug formulation linked tp natural product is allied with some inherent problems, therefore therapeutic manufacturing has erased its chief emphasis toward synthetic compound collections and high throughput screening (HTS) for new drug formulation. But consequences, did not fulfill the expectations and appeared as decreasing number of new drugs accomplishment in the market. This condition restored the awareness in natural product-based drug discovery, despite its high complexity, which in turn required a broad interdisciplinary research approach (Atanasov et al., 2015). The adaptable characters of bioactive compounds in curing the human diseases was very well documented in past with lot of challenges regarding their isolation, quantity and uses while latest development of technology open resurgence in extraction of bioactive compound as natural products followed by more valuable applications in controlling infectious diseases (Egbunaet al. 2019).

The increasing interest of the researchers in extract screening and isolation of effectives leads through advanced technologies followed by the demand of pharmaceutical industries also increase the pressure on inhabitants of wild plants of medicinal significance as overharvesting is risk for the medicinal species. Moreover, in future the bioactive compounds derived from herbal/medicinal plants will be used for new drug formulation which may lead to depletion in these plants. This is the main challenge in future for new formulation which should be addressed properly before time. The screening of extract required more appropriate techniques for the use of bioactive compounds present in it to understand the molecular basis of several chronic diseases. Anywar, \& Namukobe, (2020) reported about factors that are involved in investigations based on new drug formulation related to the plants and significant challenges that must be encountered in drug discovery from plants. The factors upsetting specially for plant-based products in drug discovery include; abundance of plant or sources material, efficacy of plant material/ compound, ease of structural modification, molecular size, toxicity, and stability of the compounds.

\section{Conclusion}


It was concluded that the bioactive compounds epitomize an appreciated resource of phytochemicals with effective antimicrobial and antioxidant activities. These are Natural products found in nature specially in plants that frequently used in pharmacological or biological activity for the formulation of drugs. As microbial resistance against synthesized antibiotics and its quick development put serious apprehension in the treatment of transferrable sicknesses. Drugs from natural origin has been found active against several chronic diseases. Herbal/medicinal plants produce a large number of diverse numbers of bioactive compounds or secondary metabolites found effective against bacteria, fungi, and plants. They are of high interest and importance because they often exhibit a broad spectrum of biological activities.

\section{Conflict of Interest}

It is declared that there is no conflict of interest in between Authors

\section{Acknowledgement}

Author acknowledge Dean faculty of Science of University of Haripur for funding of this project

\section{References}

1. Adil, I. H. (2006). Pressurized liquid extraction of phenolic compounds from fruit pomaces (Doctoral dissertation, Doctoral dissertation). Natural and Applied Sciences, Middle East Technical University. Ph. D. Thesis, 122 pp, Ankara, TURKEY.

2. Allegra, M. (2019). Antioxidant and Anti-Inflammatory Properties of Plants Extract. Antioxidants (Basel) 8(11): 549.

3. Anywar, G., \& Namukobe, J. (2020). Factors affecting the choice for plant-based products in drug discoveries. In Phytochemicals as Lead Compounds for New Drug Discovery (pp. 15-24). Elsevier.

4. Ashmawy, N. A., Salem, M. Z., Mervat, E. H., Abd El-Kareem, M. S., El-Shanhorey, N. A., Mohamed, A. A., \& Salem, A. Z. (2018). Antibacterial activity of the bioactive compounds identified in three woody plants against some pathogenic bacteria. Microbial pathogenesis, 121, 331-340.

5. Atala, E., Vásquez, L., Speisky, H., Lissi, E., \& López-Alarcón, C. (2009). Ascorbic acid contribution to ORAC values in berry extracts: An evaluation by the ORAC-pyrogallol red methodology. Food Chemistry, 113(1), 331-335.

6. Atanasov, A. G., Waltenberger, B., Pferschy-Wenzig, E. M., Linder, T., Wawrosch, C., Uhrin, P., ... \& Rollinger, J. M. (2015). Discovery and resupply of pharmacologically active plant-derived natural products: A review. Biotechnology advances, 33(8), 1582-1614.]

7. Baytop, T. (1984). Türkiyede bitkiler ile tedavi (geçmişte ve bugün) (Vol. 40). İstanbul Üniversitesi.

8. Bernardini, S., Osorio, M. S., \& Tiezzi, A. (2018). Plants: An Infinite Source of Molecules Useful for Pharmaceuticals. Current Traditional Medicine, 4(3), 157-165.

9. Breu, F., Guggenbichler S., \& Wollmann, (2008). Antimicrobial Resistance in DevelopingCountries. Springer New York Dordrecht Heidelberg London, 3-15.

10. Choi, C. W., Kim, S. C., Hwang, S. S., Choi, B. K., Ahn, H. J., Lee, M. Y., ... \& Kim, S. K. (2002). Antioxidant activity and free radical scavenging capacity between Korean medicinal plants and flavonoids by assay-guided comparison. Plant science, 163(6), 1161-1168.

11. Davies J (1994): Inactivation of antibiotics and the dissemination of resistance genes. Science 264: 375-382.

12. de Laet Santana, P., Nataly Busato deFeiria, S., \& Francisco Hofling, J. (2016). Medicinal Plants as a Source of New Therapeutic Products: Genus Mentha and the Potential Antimicrobial Activity of Extracts and Essential Oils. Current Traditional Medicine, 2(2), 94-114.

13. Dulger, B., \& Gonuz, A. (2005). Antibacterial activity of the endemic Hypericum kazdaghensis. Fitoterapia, 76(2), 237-239. 
14. Dulger, B., Gonuz, A., Bilen, S., \& Jäger, A. K. (2005). Antimicrobial studies on three Hypericum species from Turkey. South African Journal of Botany, 71(1), 100-103.

15. Egbuna, C., Kumar, S., Ifemeje, J. C., Ezzat, S. M., \& Kaliyaperumal, S. (Eds.). (2019). Phytochemicals as Lead Compounds for New Drug Discovery 2020, Pages 15-24. Elsevier

16. Entenza, J. M., Betrisey, B., Manuel, O., Giddey, M., Sakwinska, O., Laurent, F., \& Bizzinib,A. (2014). Rapid detection of staphylococcus aureus strains with reduced susceptibility to vancomycin by isothermal micro calorimetry. Journal of Clinical Microbiology, 52(1), 180-186.

17. Ercisli, S. (2007). Chemical composition of fruits in some rose (Rosa spp.) species. Food chemistry, 104(4), 1379-1384.

18. Erdogrul, Ö. T. (2002). Antibacterial activities of some plant extracts used in folk medicine. Pharmaceutical Biology, 40(4), 269-273.

19. Gupta, A., Naraniwal, M., \& Kothari, V. (2012). Modern extraction methods for preparation of bioactive plant extracts. International journal of applied and natural sciences, 1(1), 8-26.

20. Ismaili, H., Milella, L., Fkih-Tetouani, S., Ilidrissi, A., Camporese, A., Sosa, S., ... \& Aquino, R. (2004). In vivo topical anti-inflammatory and in vitro antioxidant activities of two extracts of Thymus satureioides leaves. Journal of ethnopharmacology, 91(1), 31-36.

21. Keleş, O., Ak, S., Bakirel, T., \& Alpinar, K. (2001). Screening of some Turkish plants for antibacterial activity. Turkish Journal of Veterinary and Animal Sciences, 25(4), 559-565.

22. Khameneh, B., Iranshahy, M., Soheili, V., \& Bazzaz, B. S. F. (2019). Review on plant antimicrobials: A mechanistic viewpoint. Antimicrobial Resistance \& Infection Control, 8(1), 118.

23. Muthukumran, P., Begumand, V. H., \& Kalaiarasan, P. (2011). Anti-diabetic activity of Dodonaea viscosa (L) leaf extracts. International Journal of PharmTech Research, 3(1), 136-139.

24. Nascimento, G. G.; Locatelli, J.; Freitas, P. C. and Silva, G. L. (2000). Antibacterial activity of plant extracts and phytochemicals on antibiotic-resistant bacteria. Brazilian Journal of Microbiol, 31 (4): 247-256.

25. Ozturk, S., \& Ercisli, S. (2005). Broad-Spectrum Antibacterial Properties of Thymus fallax. Pharmaceutical biology, 43(7), 609-613.

26. Relman, D. A. (2002). New technologies, human-microbe interactions, and the search for previously unrecognized pathogens. The Journal of infectious diseases, 186 (Supplement_2), S254-S258.

27. Salehi, P., Sonboli, A., Eftekhar, F., Nejad-Ebrahimi, S., \& Yousefzadi, M. (2005). Essential Oil Composition, Antibacterial and Antioxidant Activity of the Oil and Various Extracts of Ziziphora clinopodioides subsp. rigida (B OISS.) R ECH. f. from Iran. Biological and Pharmaceutical Bulletin, 28(10), 1892-1896.

28. Sokmen, A., Gulluce, M., Akpulat, H. A., Daferera, D., Tepe, B., Polissiou, M., ... \& Sahin, F. (2004). The in vitro antimicrobial and antioxidant activities of the essential oils and methanol extracts of endemic Thymus spathulifolius. Food control, 15(8), 627-634.

29. Sokmen, A., Jones, B. M., \& Erturk, M. (1999). Antimicrobial activity of extracts from the cell cultures of some Turkish medicinal plants. Phytotherapy Research: An International Journal Devoted to Pharmacological and Toxicological Evaluation of Natural Product Derivatives, 13(4), 355-357.

30. Sommer, M. O., \& Dantas, G. (2011). Antibiotics and the resistant microbiome. Current opinion in microbiology, 14(5), 556-563.

31. Subedi, L., Timalsena, S., Duwadi, P., Thapa, R., Paudel, A., \& Parajuli, K. (2014). Antioxidant activity and phenol and flavonoid contents of eight medicinal plants from Western Nepal. Journal of Traditional Chinese Medicine, 34(5), 584-590.

32. Wright, G. D. (2017). Opportunities for natural products in 21 st century antibiotic discovery. Natural product reports, 34(7), 694-701. 
33. Xiao, P., Li, X., Chen, J., \& Duan, J. A. (2017). Simultaneous Quantification of Three Chemical Types Bioactive Compounds in Radix Isatidis and Its Relevant Pharmaceutical Dosage Forms by HPLC-DAD. Journal of the Brazilian Chemical Society, 28(7), 1237-1246.

34. Yahia, Y., Benabderrahim, M. A., Tlili, N., Bagues, M., \& Nagaz, K. (2020). Bioactive compounds, antioxidant and antimicrobial activities of extracts from different plant parts of two Ziziphus Mill. species. PloS one, 15(5), e0232599.

Zheng, L., \& Dong, D. (2011). Development and Validation of an HPLC Method for Simultaneous Determination of Nine Active Components in 'Da-Chai-Hu-Tang'. Chinese Medicine, 2(01), 20.

Received: 31 ${ }^{\text {th }}$ August, 2020

Accepted: $1^{\text {th }}$ December, 2020 